\title{
ISOLATION, IDENTIFICATION, ASSESSMENT OF PATHOGENECITY AND ANTIBIOTIC SENSITIVITY OF YERSINIA ENTEROCOLITICA ISOLATES FROM PIGS
}

\author{
R. BORAL*, R. S. RATHORE, S. PAUL \\ A.K. MISHRA ${ }^{1}$ AND G. PATRA ${ }^{2}$ \\ Division of Veterinary Public Health \\ Indian Veterinary Research Institute \\ Izatnagar, Bareilly- 243 122, UP, India
}

Yersinia enterocolitica is an important zoonotic pathogen that causes yersiniosis in humans and animals. The present study was undertaken to detect $Y$. enterocolitica from pigs and foods of porcine origin by conventional cultural technique, which is not generally used owing to its cumbersome and time consuming nature. Isolation of Yersinia spp. by conventional microbiological techniques includes cold enrichment of samples for 3 weeks followed by alkaline treatment and selective isolation using specific media for Yersinia spp. like cefsulodin irgasan novobiocin (CIN) media along with Yersinia selective supplement. In vitro pathogenecity and antibiotic resistance pattern of the isolates recovered were also studied. Typical cultural and biochemical characteristics were used for the identification and confirmation of $Y$. enterocolitica isolates. Occurrence rate in pig faeces was $6.25 \%$; in pig tongues it was $7.5 \%$; in pig tonsils it was $15 \%$ and it was $5 \%$ in the ground pork samples. Occurrence rate was $\mathbf{8 \%}$ with highest occurrence found in pig tonsils, whereas lowest in the ground pork samples. All the isolates were found positive for congo red binding test, crystal violet binding test and autoagglutination tests. In vitro sensitivity profile of the isolates against 12 commonly used antibiotics revealed that $100 \%$ of $Y$. enterocolitica isolates were sensitive to amikacin and gentamycin and resistant to erythromycin and ampicillin. Most of the isolates were sensitive for ciprofloxacin $(87.5 \%)$, norfloxacin $(87.5 \%)$, neomycin $(87.5 \%)$, tetracycline $(75 \%)$, nalidixicacid (75\%).

Key words: Antibiotic sensitivity, CIN agar, Foodborne infection, Pigs, Yesinia enterocolitica

Among the members of genus Yersinia, Yersinia enterocolitica is considered as the most important foodborne pathogen, and is recognized worldwide as an emerging food borne zoonotic pathogen. Y. enterocolitica is a ubiquitous micro-organism indigenous to gastrointestinal tract of warm blooded animals like pig, dog, rodent etc. The

\footnotetext{
*Corresponding Author

${ }^{1}$ Division of Animal Health, Central Institute for Research on Goats, Makhdoom, Mathura 281122, U.P.

${ }^{2}$ Deptartment of LPT, Faculty of Veterinary and Animal Sciences, WBUAFS, Kolkata- 700037
} 
organism has been isolated from a variety of foods like raw and pasteurized milk, unchlorinated drinking water, fresh vegetables, raw meat, shellfish, beef, oysters, mussels and environmental samples (Fredriksson-Ahomaa et al., 2006; Subha et al., 2009; Virtanen et al., 2012; Paixao et al., 2013). The significance of $Y$. enterocolitica is further strengthened by its capability to multiply at refrigeration temperatures and in vaccum packed food. Being reported from above 33 countries worldwide the incidence of $Y$. enterocolitica is apparently increasing (Bottone, 1999). Pigs are reported to be a major source of Yersinia enterocolitica and harbor the organism in their throat and tonsils (Funk et al., 2000) as well as shed it in faeces (Rasmussen et al., 1995). $Y$. enterocolitica has been frequently been isolated from slaughterline pigs (Bonardi et al., 2003). Among numerous $Y$. enterocolitica serotypes, O:3, O:9, O:8, O:5 and 27 are associated with disease in humans and the main reservoir of serotype O:3 and O:9 is believed to be healthy pigs (Nesbakken et al., 2003). The disease is characterized by enteritis leading to diarrhea, abdominal pain, cramps, fever and at times vomiting in youngs. In adults, pharyngitis, terminal ileitis and pseudo appendicitis syndrome are common. Secondary immunological complications may include erythema nodosum, reactive arthritis, Reiter's syndrome, glomerulo nephritis, myocarditis or thyroiditis (Cornelis et al., 1987; Baumgartner et al., 2007). In animals, enteritis and septicaemia is commonly seen. However, many animals may remain as symptomless a carrier which is very important from public health point of view. Isolation of $Y$. enterocolitica is difficult, the current method for the isolation include enrichment followed by streaking on selective agar such as cefsulodin, irgasan and novobiocin medium (CIN) (Mehlmann et al., 1978; Schiemann, 1979). Due to the cumbersome and time consuming isolation procedure, there are fewer reports of $Y$. enterocolitica from India. The study was undertaken with the objective to isolate and identify $Y$. enterocolitica from pigs and to test the isolates for their pathogenecity and antibiotic sensitivity.

\section{MATERIALS AND METHODS}

Reference bacterial strains: The bacterial strains used in this study were obtained from IMTECH, Chandigarh (Yersinia enterocolitica MTCC 840, MTCC 859 and MTCC 861). The strains were tested for their cultural, morphological, biochemical and pathogenic characteristics. All the strains were maintained by subculturing every month in brain heart infusion (BHI) slants.

Collection and processing of samples: A total of 300 samples from various sources (Pig farm IVRI, local private pig farms and slaughter house) were collected from different farms (Pig Farm IVRI, local private pig farms) and slaughter houses (IVRI slaughter house and local slaughter houses) in and around Bareilly, Uttar 
Pradesh (Table 1). All the samples were collected in sterile conditions and transported to laboratory as quickly as possible for further processing.

Enrichment and Culturing: The fecal sample from pigs were inoculated into PBS containing $0.15 \%$ bilesalts and $1 \%$ sorbitol and incubated at $4^{\circ} \mathrm{C}$ for 3 weeks. In case of tissue samples, about $10 \mathrm{~g}$ of each tongue, tonsil and meat tissue of pig was cut into small pieces, and added in $90 \mathrm{~mL}$ PBS containing $0.15 \%$ bile salts and $1 \%$ sorbitol. The inoculated medium was then homogenized for 2 minutes in a stomacher. The homogenized medium was allowed to stand undisturbed at room temperature for 10 minutes to allow settling of large meat particles. Then the medium was incubated at $4^{\circ} \mathrm{C}$ for 3 weeks (Bercovier and Mollaret, 1984; ISO, 2003; Markey et al., 2013). Following 3 weeks of cold enrichment in PBS containing $0.15 \%$ bile salts and $1 \%$ sorbitol, the inoculum was streaked onto cefsulodin irgasan novobiocin (CIN) plates, and incubated at $22^{\circ} \mathrm{C}$ for 7 days (Schiemann,1979). Alternatively, $0.5 \mathrm{~mL}$ of enriched culture was mixed with $4.5 \mathrm{~mL}$ dilute aqueous alkali solution $(0.5 \% \mathrm{KOH}$ : $0.5 \% \mathrm{NaCl}$ ) for $15 \mathrm{sec}$ and immediately streaked onto CIN agar (Delmas and Vidon, 1985; Fukushima, 1985; Luis et al., 2013). The culture plates were checked daily for typical colonies for 7 days. Plating was also done on MacConkey agar and bismuth sulphite agar (BSA) (Cowan, 1975; Franzin et al., 1984; Stern and Oblinger, 1980).
Identification of genus Yersinia and $\boldsymbol{Y}$. enterocolitica: For confirmation of genus Yersinia Gram's staining, motility test and various biochemical reactions like oxidase test, urease test, citrate test, acid and alkali reaction on Kligle rIron agar (KIA) slants were performed (Cowan, 1975; Bercovier and Mollaret, 1984). The colonies of genus Yersinia were subjected to lysine, arginine and ornithine decarboxylase test, VogesProskauer (VP) test, methyl red test, phenylalanine (PA) deaminase test and motility for identification of $Y$. enterocolitica (Holt et al., 1994; Markey et al., 2013).

Bio-grouping of $Y$. enterocolitica: Positive cultures were subjected to nitrate reduction test, indole test, sugar utilization test, Esculin hydrolysis test, lipase test and deoxyribonuclease (DNase) test under modified Wauter's scheme for confirmation and bio-grouping of $Y$. enterocolitica (Bercovier et al., 1980; Luis et al., 2013).

In vitro pathogenicity testing and antibiotic sensitivity profiling of the isolates: Isolates were subjected to auto-agglutination in MRVP broth (Aulisio et al., 1983; Markey et al., 2013) and Congo red binding/ crystal violet binding assay (Prpic et al., 1983; Bhaduri et al., 1987; Luis et al., 2013) for testing the virulence. The recovered isolates were tested against commonly used antibiotics (Table 2) using antibiotic discs (Hi Media) according to the criteria of the National Committee for Clinical Laboratory Standards by disc diffusion technique (Bauer et al., 1966; NCCLS, 2002). 


\section{RESULTS}

Isolation studies: Among total 200 samples subjected for the bacterial isolation, 16 were found positive for $Y$. enterocolitica and the sources of the isolation are shown in Table 1.

Cultural / colony characteristics: Typical dark red colonies surrounded by transparent border; usually flat with smooth border and entire edge giving a characteristic "Bulls-Eye" appearance, were observed after $72 \mathrm{hrs}$ of incubation on CIN agar (Fig. 1A) in case of positive samples, whereas in MacConkey agar, the colonies were pale pink (Fig. 1B) and in bismuth sulphite agar they were black enamel like (Fig. 1C). Gram staining revealed gram negative coccobacillary morphology.

Table 1. Details of the samples collected and subjected for bacterial isolation

\begin{tabular}{cccc}
\hline Sl. No. & Sample type & Sample No. & $\begin{array}{c}\text { Sample positive (\%) } \\
\text { for } \text { Y. enterocolitica }\end{array}$ \\
\hline 1 & Pig faeces & 80 & $5(6.25 \%)$ \\
2 & Pig tongues & 40 & $3(7.5 \%)$ \\
3 & Pig tonsils & 40 & $6(15 \%)$ \\
4 & Pork & 40 & $2(5 \%)$ \\
\hline \multicolumn{2}{l}{ Total number of samples } & $\mathbf{2 0 0}$ & $\mathbf{1 6}(\mathbf{8 \%})$ \\
\hline
\end{tabular}

Table 2. Antibiogram of $Y$. enterocolitica isolates $(n=16)$

\begin{tabular}{|c|c|c|c|c|c|}
\hline \multirow[t]{2}{*}{ Sl. No. } & \multicolumn{2}{|c|}{ Antibiotic (Conc.) } & \multicolumn{3}{|c|}{ Antibiogram of isolates $n(\%)$} \\
\hline & & & Sensitive & Intermediate & Resistant \\
\hline 1 & Streptomycin & $(10 \mathrm{mcg})$ & $10(62.5 \%)$ & $3(18.75 \%)$ & $3(18.75 \%)$ \\
\hline 2 & Nalidixic acid & $(30 \mathrm{mcg})$ & $12(75 \%)$ & $2(12.5 \%)$ & $2(12.5 \%)$ \\
\hline 3 & Gentamycin & $(10 \mathrm{mcg})$ & $16(100 \%)$ & $0(0 \%)$ & $0(\%)$ \\
\hline 4 & Tetracycline & (30 mcg) & $12(75 \%)$ & $0(0 \%)$ & $4(25 \%)$ \\
\hline 5 & Amikacin & $(10 \mathrm{mcg})$ & $16(100 \%)$ & $0(0 \%)$ & $0(\%)$ \\
\hline 6 & Erythromycin & $(15 \mathrm{mcg})$ & $0(0 \%)$ & $0(\%)$ & $16(100 \%)$ \\
\hline 7 & Norfloxacin & (10 mcg) & $14(87.5 \%)$ & $2(12.5 \%)$ & $0(\%)$ \\
\hline 8 & Ampicillin & $(10 \mathrm{mcg})$ & $0(0 \%)$ & $0(\%)$ & $16(100 \%)$ \\
\hline 9 & Neomycin & $(30 \mathrm{mcg})$ & $14(87.5 \%)$ & $0(\%)$ & $2(12.5 \%)$ \\
\hline 10 & Cephalothin & $(30 \mathrm{mcg})$ & $0(\%)$ & $2(12.5 \%)$ & $14(87.5 \%)$ \\
\hline 11 & Ciprofloxacin & $(10 \mathrm{mcg})$ & $14(87.5 \%)$ & $2(12.5 \%)$ & $0(\%)$ \\
\hline 12 & Chloramphenicol & (30 mcg) & $10(62.5 \%)$ & $3(18.75 \%)$ & $3(18.75 \%)$ \\
\hline
\end{tabular}


Biochemical analysis and biotyping: Colonies which were citrate negative (-), urease positive (+), and gave an alkaline slant/ acid butt without gas or $\mathrm{H}_{2} \mathrm{~S}$ on KIA were used for species identification and biogrouping (Table 3). The isolates recovered under the present study were positive for catalase, glucose, maltose, mannose, sucrose, xylose, maltose, D-xylose, sorbitol, manitol, whereas rhamnose, indole, raffinose, ornithine, oxidase, citrate utilization test, lysine, arginine, they showed negative results. Esculin hydrolysis and lipase yielded variable results. On the basis of motility at $25^{\circ} \mathrm{C}$, negative citrate utilization test, positive nitrate reduction test and positive Voges-Proskauer test, the isolates were confirmed as $Y$. enterocolitica (Table 3). Most of the isolates belonged to biotype1 $\mathrm{A}$ and few belonged to biotype 3 .
In vitro pathogencity testing: In MR-VP broth, the tube incubated at lower temperature $\left(25^{\circ} \mathrm{C}\right)$ showed turbidity, whereas the tube incubated at $37^{\circ} \mathrm{C}$ showed positive agglutination of bacteria along the bottom/wall of test tube and clear supernatant fluid. Positive isolates displayed tiny red colonies and small intensely purple colonies in congo red binding test and crystal violet binding test, respectively. All the isolates were positive for congo red binding test, crystal violet binding test and autoagglutination tests.

Antibiotic sensitivity profile of $Y$. enterocolitica isolates: The isolates recovered under present study showed high sensitivity to gentamycin, amikacin, ciprofloxacin, neomycin, norfloxacin and tetracycline, whereas moderate sensitivity

\section{Table 3. Biochemical properties of $Y$. enterocolitica}

\begin{tabular}{|c|c|c|c|c|c|}
\hline $\begin{array}{c}\text { Name of the } \\
\text { biochemical } \\
\text { test }\end{array}$ & $\begin{array}{c}\text { Standard / } \\
\text { Reference } \\
\text { isolate }\end{array}$ & $\begin{array}{l}\text { Test / } \\
\text { Study } \\
\text { isolate }\end{array}$ & $\begin{array}{c}\text { Name of the } \\
\text { biochemical } \\
\text { test }\end{array}$ & $\begin{array}{l}\text { tandard / } \\
\text { Reference } \\
\text { isolate }\end{array}$ & $\begin{array}{l}\text { Test / } \\
\text { Study } \\
\text { isolate }\end{array}$ \\
\hline Catalase test & + & + & Lysine decarboxylase & - & - \\
\hline Oxidase test & _- & _ & Ornithine decarboxylase & + & + \\
\hline Indole test & + & + & Sucrose fermentation & + & + \\
\hline MR test & + & + & Rhamnose fermentation & - & - \\
\hline VP test & + & + & Raffinose fermentation & - & - \\
\hline Citrate utilization & - & - & Xylose fermentation & + & + \\
\hline Glucose fermentation & + & + & Maltose fermentation & + & + \\
\hline Sorbitol fermentation & + & + & Mannose fermentation & + & + \\
\hline Urease test & + & + & Lipase test & $+/-$ & $+/-$ \\
\hline Arginine decarboxylase & - & - & Esculin hydrolysis & $+/-$ & $+/-$ \\
\hline Trehalose fermentation & + & + & Nitrate reduction & + & + \\
\hline KIA & $+/-$ & $+/-$ & Deoxyribonuclease & - & - \\
\hline
\end{tabular}


towards streptomycin and chloramphenicol (Table 2). The isolates were resistant against ampilcillin, cephalothin and erythromycin (Table 2).

\section{DISSCUSSION}

Yersinia enterocolitica, formerly referred to as Bacterium enterocoliticum, Pasterulla pseudotuberculosis type B and Pasteurella $X$, is a ubiquitous, gram-negative bacterium pathogenic to human beings (Schleifstein and Coleman, 1943; Frederiksen, 1964). Y. enterocolitica is a psychophillic food and water borne entero-invasive pathogen causing enterocolitis, acute mesenteric lymphadenitis, mimicking appendicitis, post infectious arthritis, systemic infections, nausea, vomiting, diarrhoea, septicemia, skin and eye infection in humans (Baumgartner et al., 2007). Pigs are regarded as a major reservoir and asymptomatic carrier of $Y$. enterocolitica. In the present study, various aspects related to microbiological isolation (cultural and biochemical characterization of the isolates) of this fastidious pathogen was explored to assess the chances of food borne infection to human.

Under the present study, among 200 samples 16 were found positive for $Y$. enterocolitica. Pig tonsils (15\%) and pig tongue $(7.5 \%)$ showed maximum occurrence of infection followed by pig faeces $(6.25 \%)$ and ground pork samples (5\%). Likewise, earlier investigators have reported higher rate of prevalence of $Y$. enterocolitica in pig tonsils and tongue, while low incidence was reported in ground pork (De Boer and Nouws, 1991;
Fredriksson-Ahomaa et al., 2009; Arora, 2010; Baghel and Kumar, 2017). Arora (2010) in his study also reported higher occurrence of $Y$. enterocolitica in pig tonsils $(34.74 \%)$ as compared to ground pork $(4.35 \%)$. The variations in the occurrence may be attributed to difference in methods of sample collection, enrichment broths, enrichment methods and incubation steps used. Pigs are regarded as a major reservoir of $Y$. enterocolitica and asymptomatically carry the pathogen particularly in their tonsils and tongue. Therefore, the consumption of porcine tongue and tonsils in particular is considered a risk factor (Bottone, 1999). In case of asymptomatic carriers, pathogens from these infected tissues may contaminate the carcass during slaughter and dressing procedure (Nesbakken et al., 2003).

Isolation of $Y$. enterocolitica from food is more difficult than from clinical specimens with active infections because the pathogen may be present in low numbers, and so requires enrichment in liquid media prior to streaking on solid media (Schiemann, 1982). Nowak et al. (2006) reported that the use of PBS with $1 \%$ sorbitol and $0.15 \%$ bile salts for enrichment of samples for 2-3 weeks followed by streaking on CIN agar was the most sensitive cultural method for detection of $Y$. enterocolitica in pig fecal contents and the second most sensitive method for detection of $Y$. enterocolitica in pig tonsils. Although the method was time consuming but yielded satisfactory result under the present study. $Y$. enterocolitica appeared as 0.5 to $1.0 \mathrm{~mm}$ 
Indian Journal of Animal Health, June, 2018

Isolation, identification, pathogenecity and antibiotic sensitivity of $Y$. enterocolitica

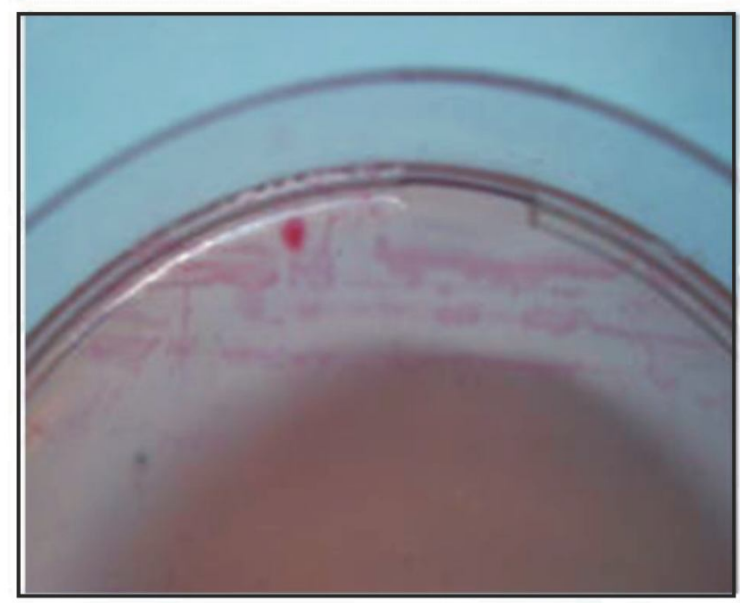

A

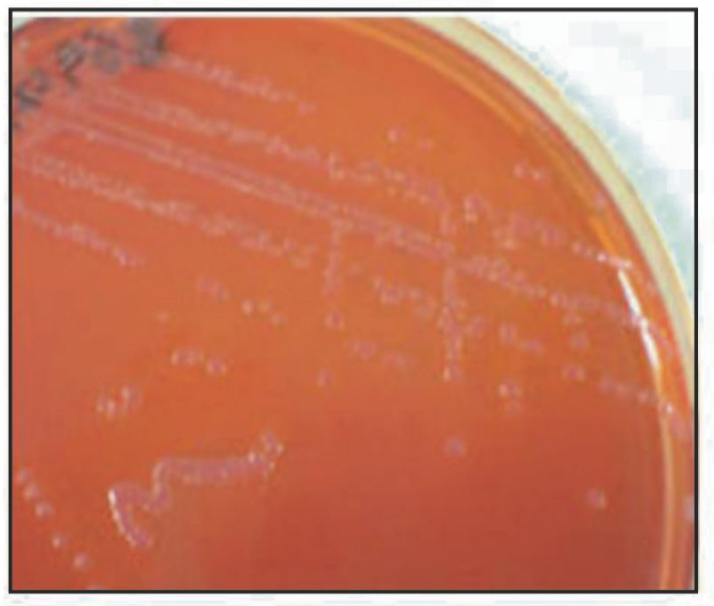

B

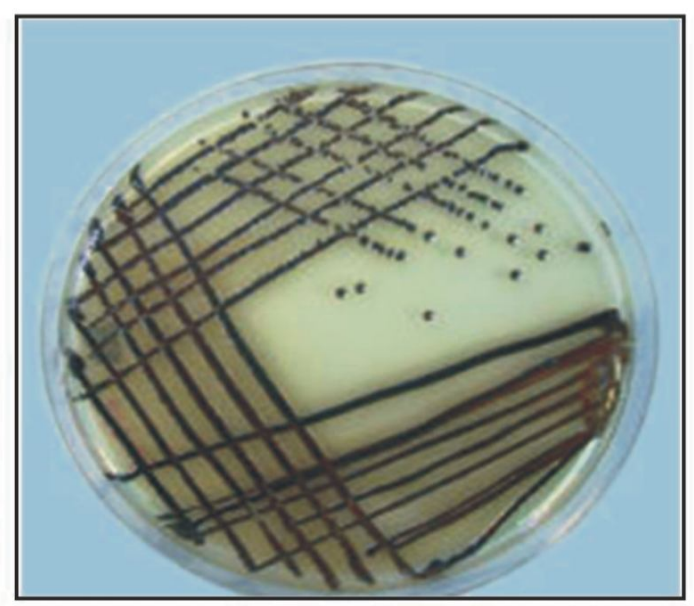

C

Fig. 1. (A) Colonies of Y. enterocolitica on CIN agar

(B) MacConkey agar Bismuth

(C) Sulphite agar plates 
colonies with a red "Bull's-Eye" and a clear border (Fig. 1A) as reported previously (Schiemann, 1979). Dark enamel like colonies (Fig. 1C) on bismuth sulphite agar were characteristic as reported by other workers (Stern and Oblinger, 1980). Although, CIN agar has been reported to be inhibitory to most of the food borne pathogens, but, some strains of Citrobacter spp. and Enterobacter spp. imitates similar colonial morphology, hence to distinguish $Y$. enterocolitica from nonyersiniae bacteria, a battery of biochemical tests were employed. The results of the biochemical tests (Table 3) confirmed the presence of $Y$. enterocolitica in the samples which were in agreement with the findings of Bercovier and Mollaret, 1984; Holt et al., 1994.

Among 200 samples, 16 isolates of $Y$. enterocolitica were recovered by cultural method. For biotyping of Y.enterocolitica, modified Wauter's scheme was adopted (Bercovier et al., 1980; Bottone, 1999). Most of the isolates belonged to biotype $1 \mathrm{~A}$ and few belonged to biotype 3 (Table 3 ). Clinical, epidemiological and experimental evidence suggests that few biotype $1 \mathrm{~A}$ strains of $Y$. enterocolitica are virulent, and can cause gastrointestinal disease (Tennant et al., 2003) and have been implicated in nosocomial (Ratnam et al., 1982) and foodborne (Greenwood and Hooper, 1990; Butt et al., 1991) outbreaks of yersiniosis. Biotype 1A strains produce heat stable enterotoxin which invades cultured epithelial cells (Grant et al., 1998), and resist killing by macrophages (Grant et al., 1999). The biotype 1A strains (serotype O: 6,30 ) have also been reported to cause placentitis and abortion in pregnant ewes (Corbel et al., 1992).

In vitro pathogenicity testing included autoagglutination testing, congo red binding and crystal violet binding. All the isolates were positive for congo red binding test, crystal violet binding test and autoagglutination tests. The results of biotyping and in vitro pathogenicity testing were comparable to that reported by other workers in India (Singh and Virdi, 1999; Singh et al., 2003).

All the strains of $Y$. enterocolitica isolates from different samples were sensitive to amikacin and gentamycin (100\%). Most of the strains were sensitive for ciprofloxacin $(87.5 \%)$, norfloxacin $(87.5 \%)$, neomycin $(87.5 \%)$, tetracycline $(75 \%)$ and nalidixicacid (75\%). Yersinia enterocolitica isolates were highly resistant to erythromycin (100\%), ampicillin (100\%) and cephalothin (90\%). Susceptibility of the isolates to amikacin and gentamycin and resistance to ampicillin, erythromycin and cephalothin were in accordance with earlier studies (Singh and Virdi, 2004; Kushal and Anand, 2001; Baumgartner et al., 2007; Subha et al., 2009).

The present study revealed that $Y$. enterocolitica are present in human food of porcine origin and therefore due precaution must be undertaken during handling of pork and raw pig tissues. Microbiological methods, although 
cumbersome and time consuming may be used for isolation and identification of the pathogen from environmental (including food) samples. Presence of biotype 1A of $Y$. enterocolitica raises a concern as it is capable of producing GI disease in human. However, more studies are required on this particular pathogen from different areas of the country for getting a complete picture of the prevalence and risk factor associated

\section{REFERENCES}

Arora D, 2010. Development of indirect-ELISA for detection of Yersinia enterocolitica in pork. M.V.Sc. thesis, Haryana Agricultural University, Hisar, India

Aulisio CCG, Stanfield JT, Hill WE and Morris JA, 1983. Pathogenicity of Yersinia enterocolitica demonstrated in the suckling mouse. J Food Prot, 46: 856-860

Baghel SS and Kumar A, 2017. Occurrence of Yersinia enterocolitica in raw pork. Haryana Vet, 56(1): 83-86

Bauer AW, Kirby WMM, Sherris JC and Turck M. 1966. Antibiotic susceptibility testing by a standardized single disc method. Am J Clin Pathol, 45: 493-496

Baumgartner A, Kuffer M, Suter D, Jemmi T and Rohner P, 2007. Antimicrobial resistance of Yersinia enterocolitica strains from human patients, pigs and retail pork in Switzerland. Int J Food Microbiol, 115: 110-114

Bercovier H and Mollaret HH, 1984. Yersinia. In Bergey's Manual of Systematic Bacteriology.Vol.1 Ed. Kreig, N.R. pp 498506. Baltimore: Williams \& Wilkins

Bercovier H, Brenner DJ, Ursing J, Steigerwalt AG with this food borne pathogen.

Conflict of interest: Authors declare that there is no conflict of interest regarding the present research work.

\section{ACKNOWLEDGEMENT}

The authors are thankful to the Director, Indian Veterinary Research Institute, Izatnagar, Bareilly for providing funds and necessary research facilities for this work.

and Fleming GR et al., 1980. Characterization of Yersinia enterocolitica senso stricto. Curr Microbiol, 4: 201-206

Bhaduri S, Conway LK and Lachica RV, 1987. Assay of crystal violet binding for rapid identification of virulent plasmid-bearing clones of Yersinia enterocolitica. J Clin Microbiol, 25: 1039-1042

Bonardi S, Brindani F, Pizzin G, Lucidi L and D'Incau M, 2003. Detection of Salmonella spp., Yersinia enterocolitica and verocytotoxin - producing Escherichia coli O157 in pig satslaughter in Italy. Int J Food Microbiol, 85: 101-110

Bottone EJ, 1999. Yersinia enterocolitica: overview and epidemiologic correlates. Microbes Infect, 1: 323-333

Butt HL, Gordon DL, Lee- Archer T, Moritz A and Merrrell WH, 1991. Relationship between clinical and milk isolates of Yersinia enterocolitica. Pathol, 23: 153-157

Corbel MJ, Ellis B, Richardson C and Bradley R, 1992. Experimental Yersinia enterocolitica placentitis in sheep. Br Vet J, 148: 339-349

Cornelis G, Laroche Y, Balligand G, Sory MP and 
Wauters G, 1987. Yersinia enterocolitica, a primary model for bacterial invasiveness. Rev Infect Dis, 9: 64-87

Cowan ST, 1975. Cowan and Steel's Manual for the Identification of Medical Bacteria, $3^{\text {rd }}$ edn., Cambridge University Press, Cambridge

De Boer E and Nouws JF, 1991. Slaughter pigs and pork as a source of human pathogenic Yersinia enterocolitica. Int J Food Microbiol, 12: $375-378$

Delmas CL and Vidon DJM, 1985. Isolation of Yersinia enterocolitica and related species from foods in France. Appl Environ Microbiol, 50: 767-771

Franzin L, Fantino P and Vidotto V, 1984. Isolation of Yersinia enterocolitica and Yersinia enterocolitica-like organisms from raw milk in Italy. Current Microbiol, 10: 357-360

Frederiksen W, 1964. A study of some Yersinia pseudotuberculosis-like bacteria ('Bacterium enterocoliticum” and Pasterulla X'). Scand Congr Pathol Microbiol Proc, 14: 103-104

Fredriksson-Ahomaa, M, Stolle A and Stephan R, 2006. Prevalence of pathogenic Yersinia enterocolitica in pigs slaughtered at a sures abattoir. Int J Food Microbiol, 119: 207-212

Fredriksson-Ahomaa M, Wacheck S, Koenig M, Stolle A and Stephan R, 2009. Prevalence of pathogenic Yersinia enterocolitica and Yersinia pseudotuberculosis in wild boars in Switzerland. Int J Food Microbiol. 135: 199202

Fukushima H, 1985. Direct isolation of Yersinia enterocolitica and Yersinia pseudotuberculosis from meat. Appl Environ Microbiol, 50: 710-712

Funk JA, Troutt HF, Davis SA and Fossler CP, 2000. In vitro susceptibility of Yersinia enterocolitica isolated from the oral cavity of swine. J Food Prot, 63: 395-399
Grant T, Bennett-Wood V and Robins-Browne RM, 1999. Characterization of the interaction between Yersinia enterocolitica biotype1A and phagocytes and epithelial cells in vitro. Infect Immun, 67: 4367-4375

Grant T, Bennett-Wood V and Robins-Browne RM, 1998. Identification of virulence-associated characteristics in clinical isolates of Yersinia enterocolitica lacking classical virulence markers. Infect Immun, 66: 1113-1120

Greenwood MH and HooperWL, 1990. Excretion of Yersinia spp. associated with consumption of pasteurized milk. Epidemiol Infect, 104: 345-350

Holt JG, Krieg NR, Sneath PHA, Staley JT and Williams ST, 1994. Bergey's Manual of Determinative Bacteriology ( $9^{\text {th }}$ edn.), Williams and Wilkins, Baltimore

ISO, 10273, 2003. Microbiology of Food and Animal Feeding Stuffs - Horizontal Method for the Detection of Presumptive Pathogenic Yersinia enterocolitica (ISO 10273:2003). International Organization for Standardization, Geneva, Switzerland

Kushal R and Anand SK, 2001. Isolation, biochemical characterization and antibiotic susceptibility of Yersinia enterocolitica isolates from milk. J Food Sci Technol, 38: 129-134.

Luis M, Maza De La, Pezzlo MT, Shigei JT, Tan GL and Peterson EM, 2013. Color Atlas of Medical Bacteria. ASM Press, Washington, D.C (USA)

Markey B, Leonard F, Archembault M, Cullinane A and Maguire D, 2013. Clinical Veterinary Microbiology. Mosby Elsevier, New York (USA)

Mehlmann, IJ, Ausilio CCG and Sanders AC, 1978. Problems in the recovery and identification of Yersinia from foods. J Assoc Anal Chem, 61: 761-771 
NCCLS, 2002. Performance standards for antimicrobial testing; twelfth international supplement. NCCLS document M100-S12. NCCLS, Wayne. ISBN: 1-56 238-454-6

Nesbakken T, Eckner K, Hoidal H and Rotterud O, 2003. Occurrence of Yersinia enterocolitica and Campylobacter spp. in slaughter pigs and consequences for meat inspection, slaughtering and dressing procedures. Int $\mathrm{J}$ Food Microbiol, 80: 231-240

Nowak B, Mueffling T, Caspari K and Hartung J, 2006. Validation of a method for the detection of virulent Yersinia enterocolitica and their distribution in slaughter pigs from conventional and alternative housing systems. Vet Microbiol, 117: 219-228

Paixao R, Moreno LZ, Gobbi DDS, Raimund, DC and Hofer E et al., 2013. Characterization of Yersinia enterocolitica biotype 1A strains isolated from swine slaughterhouses and markets. Scientific World J: 1-6

Prpic JK, Robins- Browne RM and Davey RB, 1983. Differentiation between virulent and avirulent Yersinia enterocolitica isolates using Congo red agar. J Clin Microbiol,19: 446

Rasmussen HN, Rasmussen, OF, Christensen H and Olsen JE, 1995. Detection of Yersinia enterocolitica $\mathrm{O}: 3$ in fecal samples and tonsil swabs from pigs using IMS and PCR. J Appl Bacteriol, 78: 563-568

Ratnam S, Mercer E, Picco B, Parsons S and Butler $\mathrm{R}, 1982$. A nosocomial outbreak of diarrhoel disease due to Yersinia enterocolitica serotype O:5, biotype 1A. J Infect Dis, 145: 242-247

Schiemann DA, 1979. Synthesis of a selective agar medium for Yersinia enterocolitica. Can J Microbiol, 25: 1298-1304
Schiemann DA, 1982. Development of a two-step enrichment procedure for recovery of Yersinia enterocolitica from food. Appl Environ Microbiol, 43: 14-27

Schleifstein JI and Coleman MB, 1943. An unidentified microorganisms resembling $B$. lignieri and Past. pseudotuberculosis and pathogenic for man. NY State J Med, 39: 1749-1753

Singh I and Virdi JS, 2004. In vitro antibiotic susceptibilities of Yersinia enterocolitica biotype 1A. World J Microbiol Biotechnol, 20: 329-331

Singh I and Virdi JS, 1999. Isolation, biochemical characterization and in vitro tests of pathogenicity of Yersinia enterocolitica isolated from pork. Curr Sci, 77: 1019-1021

Singh I, Bhatnagar S and Virdi JS, 2003. Isolation and characterization of Yersinia enterocolitica from diarrhoeic human subjects and other sources. Curr Sci, 84: 1353-1355

Stern NJ and Oblinger JL, 1980. Recovery of Yersinia enterocolitica from surfaces of inoculated hearts and livers. J Food Prot, 43: 706-708

Subha B, Ramakrishnan D and Suganthi V, 2009. Antimicrobial resistance pattern of selected Yersinia enterocolitica isolates from raw cow milk and pork samples of Namakkal dist. Tamilnadu, South India. Global J Environ Res, 3: $169-177$

Tennant SM, Grant TH and Robins-Brown GRM, 2003. Pathogenicity of Yersinia enterocolitica biotype 1A. FEMS Immun Med Microbiol., 38: $127-137$

Virtanen S, Salonen L and Korkeala H, 2012. Piglets are a source of pathogenic Yersinia enterocolitica on fattening-pig farms. Appl Environ Microbiol, 78: 3000-3003 